\title{
Lokale-Agenda-21-Prozesse: förderliche und hinderliche Bedingungen
}

\author{
Seit der Einführung der Lokalen Agenda 21 haben \\ sich mittlerweile über 10.000 Kommunen der \\ Handlungsinitiative angeschlossen. Bei der \\ Umsetzung treten jedoch oftmals Komplikationen \\ auf. Die Anwendung der qualitativen Meta- \\ Analyse identifiziert die hinderlichen wie förder- \\ lichen Faktoren für solche Prozesse auf lokaler \\ Ebene. Von Julia Schnepf und Norbert Groeben
}

\section{Inhaltliche Problemstellung: Nachhaltigkeitsbewusstsein im Wandel}

Im Laufe der letzten drei Jahrzehnte hat sich der Nachhaltigkeitsbegriff $\mathrm{zu}$ einem Konzept von zunehmender gesellschaftlicher Relevanz entwickelt. Das Bewusstsein um die Begrenztheit natürlicher Ressourcen hat sich sowohl bei politischen Entscheidungsträgern als auch innerhalb weiter Teile der Gesellschaft gefestigt. So zeigen etwa Umfrageergebnisse der in Deutschland durchgeführten Umweltbewusstseinsstudie aus dem Jahr 2014, dass 19 Prozent der befragten Personen $(\mathrm{N}=2.098)$ den Umweltschutz als eines der zwei wichtigsten Probleme der Bundesrepublik auffassen. Dem entsprechen die Entwicklungen im Regulierungsgrad von umwelt- und nachhaltigkeitsrelevanten Politikbereichen. So erreichte die Gesamtanzahl an Rechtsvorschriften im Geschäftsbereich des Bundesministeriums für Umwelt, Naturschutz, Bau und Reaktorsicherheit im Jahr 2017 einen neuen Höchstwert von insgesamt 235 Rechtsvorschriften. Ähnliche Entwicklungen zeichnen sich bereits seit einiger Zeit auf supranationaler Ebene ab. Heute gehen aus den Umweltaktionsprogrammen der EU in der Regel rechtlich verbindliche Richtlinien hervor und weisen damit auf eine ebenfalls zunehmende Verregelung von Umweltbelangen auf EU-Ebene hin. Als ein entscheidender Antrieb der gesamten (internationalen) Nachhaltigkeitsdebatte gilt dabei das auf dem Rio-Gipfel 1992 eingeführte Lokale-Agenda-21-Handlungsprogramm der Vereinten Nationen. Im Gegensatz zu verbindlichen Abkommen und Konventionen setzt dieses Konzept auf eine freiwillige Verpflichtung und Aktivität im Bereich der nachhaltigen Entwicklung. Mit dem Leitsatz „Global denken lokal handeln" hat es zum Ziel, Nachhaltigkeitsprozesse im lokalen Umfeld (Kommunen und Städte) zu initiieren und damit das Nachhaltigkeitsbewusstsein zu beeinflussen und ein entsprechendes Handeln der Bürger/innen langfristig zu erhal- ten. Teilnehmende Kommunen entwickeln dabei lokale Handlungspläne, um individuell festgelegte Nachhaltigkeitsziele zu erreichen. Die gewählten Mittel zur lokalen Umsetzung der Nachhaltigkeitsstrategien variieren zwischen den Kommunen jedoch stark. Ein Überblick zu konkreteren Fragen der Zielerreichung und Effizienz von Lokale-Agenda-21-Prozessen fehlt allerdings bisher (Nolte 2006), obwohl es durchaus Forschung zu diesem Problem gibt. Das liegt vor allem daran, dass die vorhandenen Studien meist qualitativer Art (mit Interview-Daten etc.) sind, deren Zusammenführung schwieriger ist als bei quantitativen Studien mit einer übereinstimmenden quantitativen Auswertungsstruktur (statistische Kennwerte, Signifikanzen, Effektstärken). Trotzdem lässt sich eine zum eingeführten Instrument der quantitativen Meta-Analyse parallele Vorgehensweise entwickeln und einsetzen: die qualitative Meta-Analyse, die von der Grundstruktur her bislang vor allem in der psychologischen Therapieforschung expliziert worden ist (vgl. Timulak 2009, Timulak/Creaner 2013, Timulak/McElvany 2013), sich aber mit Gewinn auch im Bereich ökonomie- und politikwissenschaftlicher Forschung anwenden lässt.

Das Beispiel der Lokale-Agenda-21-Prozesse stellt dabei einen besonders aussagekräftigen Testfall dar, weil es zwar ein einheitliches Leitziel gibt, nämlich dass die teilnehmenden Kommunen bei der Umsetzung der lokalen Handlungspläne eine möglichst hohe Integration der Zivilgesellschaft ermöglichen und Belange der Bürger/innen sowie diejenigen wirtschaftlicher Akteure maßvoll in die Nachhaltigkeitsplanung aufnehmen. Zugleich unterscheiden sich die teilnehmenden Kommunen aber deutlich in ihrer Größe, ihrer geopolitischen Lage und Bedeutsamkeit, ihrer wirtschaftlichen Relevanz und ihrer sozio-kulturellen Zusammensetzung. Unter Rückgriff auf vorhandene qualitative Studien sollen daher mit dem Instrument der qualitativen Meta-Analyse systematisch förderliche und hinderliche Faktoren der Lokale-Agenda-21-Prozesse identifiziert werden.

\section{Datengrundlage}

Wie bei jeder Meta-Analyse geht es darum, einen möglichst vollständigen Pool der thematisch einschlägigen Forschungsarbeiten zu identifizieren, die dann systematisch zusammengefasst werden: im Fall der qualitativen Meta-Analyse durch die Generierung aussagekräftiger Kategorien (s. u.). Für die Suche nach den relevanten Publikationen wurde auf die Recherchedatenbank Web of Science (www.apps.webofknowledge.com) zurückgegriffen. Hierbei wurden unter dem Schlagwort „lo- 


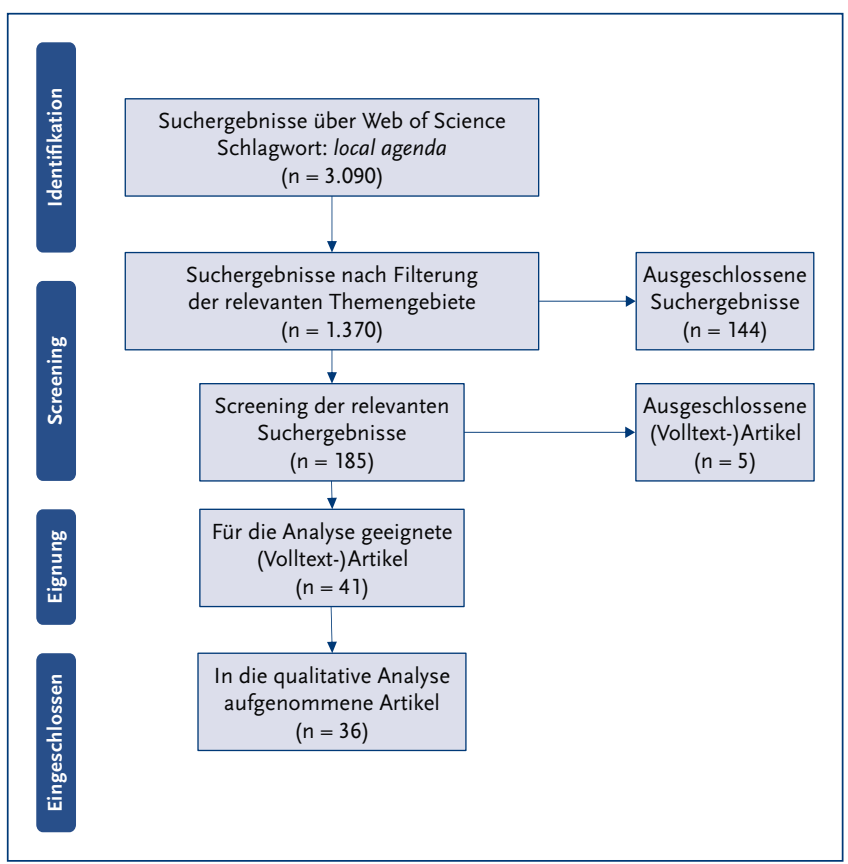

Abbildung 1: PRISMA-Flow-Diagramm: Systematische Literaturrecherche zum Thema "local agenda“

Quelle: Screening-Verfahren in Anlehnung an Moher et al. (2009)

cal agenda" in einem Zeitraum von 1992 bis 2017 insgesamt 3.090 Artikel ausfindig gemacht (siehe PRISMA-Flow-Chart, Abb.1). Dieser Suchbegriff wurde gewählt, um eine möglichst hohe Auswahl an Artikeln zu generieren. Als weitere Suchbedingung wurden für die Literaturrecherche ausschließlich (Zeitschriften-)Artikel aus wissenschaftlichen Zeitschriften mit Social-Science-Citation-Index-Eintrag gesichtet, um die Einhaltung methodisch-kontrollierter Erkenntnisgewinnung der analysierten Artikel bereits vor der qualitativen Analyse sicherzustellen. Nach diesem ersten Schritt wurde die vorhandene Auswahl an 3.090 Suchergebnissen über die Einengung auf nachhaltigkeitsrelevante Themengebiete auf 1.370 Artikel reduziert. Die weitere systematische und inhaltsgeleitete Reduktion dieser Textkorpora verlief in Anlehnung an die PRISMA-Empfehlungen zur Durchführung systematischer Literaturanalysen (Moher et al. 2009). Dabei wurden Artikel in Themenbereichen wie etwa Environmental Studies aufgenommen, während jene nachhaltigkeitsfernerer Bereiche wie Computer Science oder History ausgeschlossen wurden (siehe Tab. 3). In einem nächsten Schritt wurde das Korpus vorhandener Literaturvorschläge auf diejenigen Artikel begrenzt, die bereits mindestens einmal zitiert wurden. Für das nachfolgende Screening kamen damit 185 Artikel infrage.

Im Screening dieser 185 Artikel wurden die Inhalte der jeweiligen Abstracts untersucht. Da Lokale-Agenda-21-Prozesse im Fokus des Interesses standen, wurden nur solche Artikel ausgewählt, welche sich spezifisch auf die lokale Umsetzung von Lokale-Agenda-21-Zielen bezogen. Artikel wurden auch dann aufgenommen, wenn sie Lokale-Agenda-21-Prozesse aus einer vergleichenden Perspektive analysierten oder etwa wenn ebensolche Prozesse im Rahmen einer Indikatoren-Validierung beleuchtet wurden. Diese inhaltliche Abstract-Analyse führte zu einer weiteren Reduktion des Datensatzes auf insgesamt 41 (Volltext-)Artikel. Nach der Rezeption dieser 41 (Volltext-)Artikel mussten weitere fünf Artikel aus dem Datensatz entfernt werden, da sie Lokale-Agenda-21-Prozesse nur marginal behandelten und somit keine Information über förderliche und hinderliche Faktoren von Lokale-Agenda-21-Prozessen lieferten. Dies führte schließlich zu einer Datengrundlage von 36 analyserelevanten (Volltext-)Artikeln. Der Publikationszeitraum der ausgewählten Artikel liegt zwischen 1996 und 2015. Sieben der 36 Artikel wurden im Jahr 1998 veröffentlicht. Hier liegt die Vermutung nahe, dass besonders in diesem Zeitraum erste Evaluationsversuche von Lokale-Agenda-21-Prozessen stattfanden. Bezüglich der Referenzorte der Artikel ist eine hohe Varianz aufzufinden (vgl. Tab. 1).

Die in die Analyse eingehenden 36 (Volltext-)Artikel decken also alle übergeordneten Nachhaltigkeitsbereiche: Ökologie, Ökonomie und Soziales ab.

\section{Methode: Qualitative Meta-Analyse}

Das Zentrum einer qualitativen Meta-Analyse bildet die Umsetzung der jeweiligen qualitativen Forschungsergebnisse in für alle Studien aussagekräftige Kategorien, die den übereinstimmenden Gehalt der vorliegenden Arbeiten repräsentieren (Timulak 2009, Timulak/Creaner 2013). Diese Kategorien-Explikation sollte nicht nur aus einer rein induktiven, heuristischen Interpretation der Ausgangsuntersuchungen bestehen; bei einer solchen Konzentration auf heuristische Interpretation ist die Bezeichnung Meta-Synthese adäquater (und eingeführt: Walsh et al. 2005). Für eine meta-analytische Kategorienexplikation ist eine gleichgewichtig von allen Arbeiten und der zentralen Forschungsfrage ausgehende Extraktion relevanter inhaltlicher Aspekte wie bei einer (qualitativen) Inhaltsanalyse angezeigt (vgl. Timulak 2009, Timulak/McElvany 2013). Allerdings beziehen sich die bisher vorliegenden Beispiele qualitativer Meta-Analysen in der Psychotherapie auf eine so geringe Anzahl von Ausgangsstudien, dass zum Beispiel der objektivere Einsatz computergestützter Inhaltsanalysen nicht möglich beziehungsweise sinnvoll war. Der hier vorliegende Pool von 36 Volltexten erlaubt es aber nun, an dieser Stelle über die bisherige Entwicklung der qualitativ meta-analytischen Methodik hinauszugehen und eine computergestützte Inhaltsanalyse einzusetzen.

Hierzu wurde auf das Analyseprogramm MAXQDA zurückgegriffen, das eine explorative Inhaltsanalyse darstellt. Während innerhalb primär deduktiver inhaltsanalytischer Verfahren Kategoriensysteme in der Regel einer inhaltlichen Ableitung aus den Forschungshypothesen entsprechen (Rustemeyer 1992), bietet das Verfahren der explorativen inhaltsanalytischen Auswertung vor allem die Möglichkeit, einzelne Kodierungen (Zuordnungen zu Kategorien) induktiv aus den analyserelevanten Texten zu gewinnen (Bortz et al. 2007). Dieser Vorgang 


\begin{tabular}{|c|c|c|c|}
\hline Bezugsnation & Anzahl & Nachhaltigkeitsbereich & Anzahl \\
\hline England & 5 & Recycling \& Abfall & 7 \\
\hline Peru & 4 & Trinkwasser & 7 \\
\hline Italien & 3 & Gesundheit & 7 \\
\hline Schweden & 3 & Nachhaltige Landwirtschaft & 5 \\
\hline Spanien & 2 & $\begin{array}{l}\text { Aufenthalts- und Ausbildungsangebote } \\
\text { (für Jugendliche) }\end{array}$ & 5 \\
\hline Südafrika & 2 & (Öffentlicher) Umwelt- und Naturschutz & 5 \\
\hline Kenia & 2 & $\mathrm{CO}_{2}$-Ausstoß & 4 \\
\hline Australien & 1 & Ressourcenschonung & 4 \\
\hline Polen & 1 & (Lokale) Arbeitslosigkeitsbekämpfung & 3 \\
\hline Türkei & 1 & Biodiversität & 3 \\
\hline Tschechische Republik & 1 & Fischfang & 3 \\
\hline Senegal & 1 & Erneuerbare Energien & 3 \\
\hline Tansania & 1 & Chemikaliensicherheit & 1 \\
\hline Niederlande & 1 & Nachhaltige Forstwirtschaft & 1 \\
\hline Deutschland & 1 & Nachhaltiges Investment & 1 \\
\hline Neuseeland & 1 & & \\
\hline China & 1 & & \\
\hline Marokko & 1 & & \\
\hline Vietnam & 1 & & \\
\hline Kolumbien & 1 & & \\
\hline Baltikum & 1 & & \\
\hline EU-Vergleich & 3 & & \\
\hline
\end{tabular}

Tabelle 1: Bezugsnationen und Nachhaltigkeitsbereiche der analyserelevanten Literatur

as: Limited municipal budgets which do not allow the implementation of existing investment proposals." (Steinberg/Miranda 2005, 167). Da Ressourcenknappheit und Budgetrestriktionen in den betreffenden peruanischen Kommunen als Hemmnisse von Lokale-Agenda-21-Prozessen aufgeführt werden, wurde dieser Sinnabschnitt zunächst der Kategorie „finanzielle Ressourcen“ zugeteilt, während die von den Autoren vorgenommene Bewertung (in diesem Falle: Monieren einer $\mathrm{zu}$ geringen finanziellen Handlungsfähigkeit) in einem folgenden Schritt mit der Einteilung in die Kategorie „hinderlich“ erfasst wurde.

Im Anschluss an diesen Identifikationsprozess wurden die jeweiligen Artikel hinsichtlich ihrer Erfolgseinschätzung der behandelten Lokale-Agenda-21-Prozesse kategorisiert. Hierbei wurde eine drei stufige Likert-Skala von $1=$ „Nicht erfolgreich“, 2 = „Mittlerer Erfolg“ bis 3 = „Sehr erfolgreich“ verwendet. Als Beispiel der Kategorie „Mittlerer

ähnelt in Teilen dem durch die Chicagoer Schule propagierten Verfahren der offenen Kodierung im Sinne der Grounded Theory (Glaser et al. 1967, Strauss 1987), hat allerdings im Unterschied nicht das Ziel, in mehreren aufeinanderfolgenden Analyseschritten konkrete Zusammenhänge zwischen den einzelnen Kernkategorien zu theoretischen Aussagen zu formen.

Vielmehr liegt der Fokus der hiesigen Analyse auf einer einfachen Identifikation förderlicher und hinderlicher Faktoren bei der Umsetzung lokaler Nachhaltigkeitsprozesse. Dafür wurden sowohl Wörter als auch Sätze als Kodiereinheit verwendet. Einzelne Kategorien wurden folglich von den jeweils als förderlich oder hinderlich beschriebenen Indikatoren abgeleitet. So bildete sich beispielsweise die Kategorie der finanziellen Ressourcen aus einzelnen Nennungen der zu Verfügung stehenden (kommunalen) Mittel oder anderweitiger finanzieller Unterstützungen bei der Umsetzung der Lokale-Agenda-Projekte. Wurde die Höhe ebendieser Ressourcen in den betreffenden Artikeln, die eine solche Nennung enthielten, positiv bewertet, erhielt die Zuteilung zusätzlich die Kodierung „förderlich“ und vice versa. Als Kodierbeispiel soll hier exemplarisch die Bewertung der Rolle finanzieller Ressourcen durch Steinberg und Miranda (2005) innerhalb ihrer Analyse peruanischer Lokale-Agenda-21-Kommunen veranschaulicht werden. In ihrer Untersuchung konnten die Autoren fehlende finanzielle Mittel innerhalb der Kommunen als ein wesentliches Hindernis bei der Umsetzung lokaler Nachhaltigkeitsprogramme identifizieren: „The situation of Peruvian municipalities during the last years has meant working with an extreme resource crunch. [...] The problem of municipalities can be generalized
Erfolg“ kann hier die Bewertung des Lokale-Agenda-21-Erfolgs in Rufisque (Senegal) durch Gaye, Diouf und Keller (2001) angeführt werden. Trotz geringer Einschränkungen konstatieren die Autoren bemerkenswerte Effekte der initiierten LokaleAgenda-21-Prozesse:

„Overall, conditions are favourable for the emergence of a successful LA21 in Rufisque provided certain economic and institutional constraints are addressed. [...] On the negative side, there have been a variety of organizational problems, often compounded by bureaucratic problems, poor coordination and a general lack of resources. [...] On the positive side, however, many problems are being addressed. Workers are being trained, there are regular fora involving communities, groups, the municipality, Enda and others, and there is an increasing sense of partnership among the different stakeholders." (Gaye et al. 2001, S. 213)

Da neben positiven Formen der Zielerreichung auch unerfüllte Aspekte bei der Zielerreichung und Evaluation genannt werden, wurde dieser Sinnabschnitt mit einer Ausprägung von 2 - also „mittlerem Erfolg“ - kodiert. Die zuvor als (quantitativ) besonders bedeutsam identifizierten förderlichen und hinderlichen Faktoren wurden sodann zu den jeweiligen Erfolgseinschätzungen in Relation gesetzt.

\section{Ergebnisse}

Die computergestützte Inhaltsanalyse führt zu Kategorien, die auch eine quantitative (Häufigkeits-)Analyse ermöglichen, die eine hohe Relevanz der jeweiligen kommunalen Startbedin- 


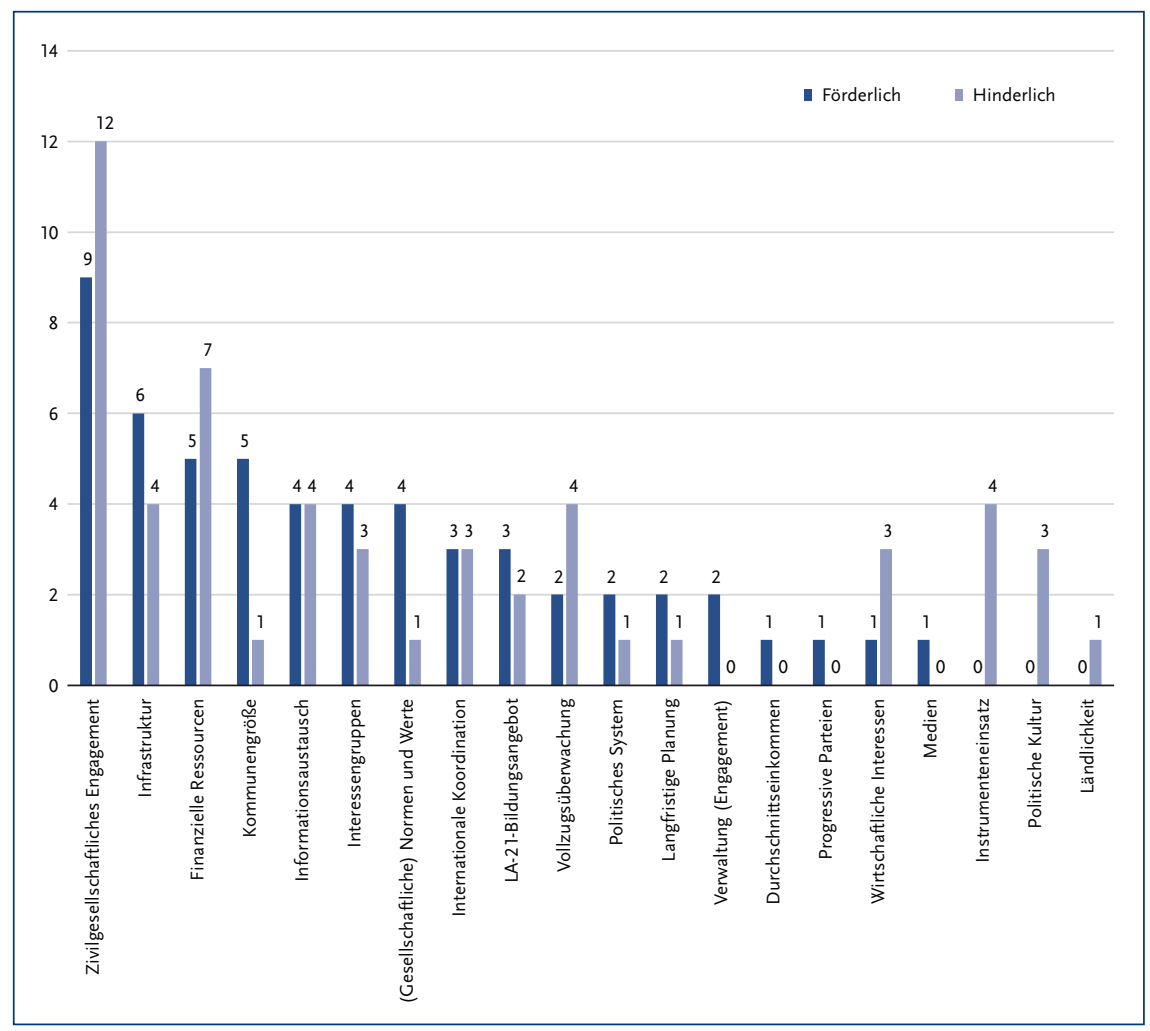

Abbildung 2: Förderliche und hinderliche Faktoren von Lokale-Agenda-21-Prozessen Anmerkung: Aufgrund von Vergleichsstudien sind Dopplungen möglich. Kodierungen sind jedoch nur einmal pro Untersuchungseinheit (z. B.: Kommune) eingegangen.

gungen bei der Umsetzung der Lokale-Agenda-21-Prozesse erkennen lassen. Es konnten insgesamt 20 relevante Faktoren bei der Umsetzung lokaler Nachhaltigkeitsziele identifiziert werden. Dabei entspricht die Faktorenstruktur in der Regel dem Prinzip einer bipolaren Variablen, sodass hohe Ausprägungen eines Faktors in der analysierten Literatur gewöhnlich mit positiven Konnotationen versehen waren, während deren negativ konnotierte Nennung als Kritik einer (zu) geringen Ausprägung des jeweiligen Faktors zu verstehen ist.

So wurden beispielsweise hohe finanzielle Ressourcen in fünf Fällen (Referenzuntersuchungseinheiten) als förderlich bewertet, während mangelnde finanzielle Ressourcen in sieben Fällen als hinderlich bewertet wurden (vgl. Abb. 2). Ledig-

\begin{tabular}{lll}
\hline Kommunale Ausgangslage & Prozessmerkmale & Zielverfolgung \\
\hline Finanzielle Ressourcen & Internationale Kooperation & Instrumenteneinsatz \\
\hline Infrastruktur & Informationsaustausch & Langfristige Planung \\
\hline Ländlichkeit & Verwaltungsengagement & Vollzugsüberwachung \\
\hline Kommunengröße & Progressive Parteien & \\
\hline Durchschnittseinkommen & LA-21-Bildungsangebot & \\
\hline Wirtschaftliche Interessen & Zivilgesellschaftl. Engagement & \\
\hline Politisches System & Interessengruppen & \\
\hline Politische Kultur & Medien & \\
\hline Gesellschaftl. Normen und Werte & \\
\hline
\end{tabular}

Tabelle 2: Übergeordnete Faktorenkategorien lich die Faktoren Verwaltungsengagement, Durchschnittseinkommen, progressive Parteien und Medien wurden ausschließlich im positiven Sinne genannt, während (ein $\mathrm{zu}$ geringer) Instrumenteneinsatz, eine hinderliche politische Kultur sowie die Ländlichkeit der Region ausschließlich mit negativen Konnotationen genannt wurden.

Wie Abbildung 2 zudem verdeutlicht, spielen nach Bewertung der jeweiligen Autoren vor allem die kommunalen Ausgangsbedingungen bei der Umsetzung lokaler Nachhaltigkeitspläne eine zentrale Rolle. Inhaltlich lassen sich die identifizierten Faktoren jedoch in drei übergeordnete Kategorien einordnen: Kommunale Ausgangslage, Prozessmerkmale und Merkmale der Zielverfolgung (vgl. Tab. 2).

Bezüglich der lokalen Ausgangslage sind es vor allem finanzielle Rahmenbedingungen wie etwa kommunale Budgetrestriktionen, die die Umsetzung der Lokale-Agenda-21-Vorhaben behindern. Exemplarisch unterstreichen - innerhalb der analysierten Literatur - etwa García-Sánchez und Lorenzo (2009) in ihrer vergleichenden Analyse zur Umsetzung der Lokale-Agenda-21-Pläne die Notwendigkeit einer soliden finanziellen Basis der Kommunen. Auch Variablen wie Kommunengröße, Infrastruktur und die Ländlichkeit der Region wurden als relevante Einflussgrößen benannt. So zeigen etwa die Ergebnisse einer kommunenvergleichenden Analyse von Llamas-Sánchez et al. (2010) in Spanien, dass Kommunen ab einer Einwohnerzahl von 50.000 deutliche Vorteile bei der Umsetzung lokaler Aktionsprogramme haben. In Bezug auf die Verbreitung nachhaltigen und regionalen Konsums zeigte sich weiter, dass vor allem vorherrschende gesellschaftliche Norm- und Wertedispositionen der lokalen Bevölkerung eine bedeutsame Rolle spielten sowie auch die verfügbaren Durchschnittseinkommen der Bürger/innen (García-Sánchez et al. 2009, Harrison et al. 1998).

Die zweite zentrale Oberkategorie der identifizierten Faktoren lässt sich unter dem Begriff Prozessmerkmale zusammenfassen. Hier erwies sich der Faktor zivilgesellschaftliches Engagement als bedeutsamste Variable. Dass ohne ein solches Engagement kaum ein Wandel hin zu nachhaltigeren Verhaltensweisen stattfindet, zeigt sich übergreifend über Regionen und Nachhaltigkeitsbereiche der analysierten Studien. Dabei fallen 
die Einbindung zivilgesellschaftlicher Akteure sowie die Umsetzung von kommunale/n Nachhaltigkeitsprojekte/n häufig leichter, wenn Lokale-Agenda-21-Bildungsangebote (Foronda 1998, Jörby 2002) gefördert werden, Interessengruppen (Nurick et al. 1998, Steinberg et al. 2005) und (als progressiv bezeichnete) Parteien die gemeinsamen Projekte vorantreiben (Echebarria et al. 2009) sowie Medien (Llamas-Sánchez et al. 2010) an der Verbreitung der Projekte mitwirken. Neben dieser zivilgesellschaftlichen Eigenschaft erweisen sich auch Merkmale der politischen Vernetzung sowie der lokalen Verwaltungseinrichtung als einflussreich. Im Sinne des Policy-Learnings und -Transfers zeigt sich beispielsweise, dass (international) vernetzte Kommunen erfolgreiche Strategien aus anderen Lokale-Agenda-21-Kommunen adaptieren können (Joas et al. 2001). Zudem erweisen sich engagierte Verwaltungsmitarbeiter/innen als förderliche Schlüsselfiguren bei der Umsetzung lokaler Handlungspläne (Tuts 1998, Banas 2010).

In einer dritten Oberkategorie wurden zudem spezifische Merkmale der Zielverfolgung zusammengefasst. Insbesondere ein zu geringer und zu wenig verbindlicher Instrumenteneinsatz wurde in einigen Fällen als hinderlicher Faktor bei der Umsetzung lokaler Nachhaltigkeitspläne bewertet (Laituri 1996, Jörby 2002, Garía-Sánchez et al. 2009). Besonders positiv beschrieben wurden hingegen langfristige Planungen sowie regelmäßige Monitoringprozesse (Vollzugsüberwachung: Velásquez 1998, Roberts et al. 2002, Echebarria et al. 2004).

Insgesamt deuten die Ergebnisse einerseits auf die Vielschichtigkeit und Komplexität der Lokale-Agenda-21-Prozesse sowie andererseits auf die Notwendigkeit unterstützender kommunaler Start- und Prozessbedingungen hin. Um abschließend den Einfluss der zwei am häufigsten genannten Faktoren auf die Erfolgsbewertung lokaler Nachhaltigkeitsprogramme zu überprüfen, wurde der statistische Zusammenhang zwischen der Nennung dieser Faktoren (zivilgesellschaftliches Engagement, finanzielle Ressourcen) und der jeweils in den untersuchten Artikeln vorgenommen Bewertung der Lokale-Agenda-21-Prozesse (Kodierung: 1 = "Nicht erfolgreich“, $2=$ „Mittlerer Erfolg“ bis 3 = „Sehr erfolgreich“) ermittelt. Hier zeigte sich, dass beide Faktoren bei positiver Ausprägung einen signifikanten Effekt auf die vorgenommene Erfolgsbewertung innerhalb der Artikel hatten. Studien, welche Kommunen mit besonders hohen finanziellen Ressourcen behandelten, berichteten folglich deutlich häufiger von geglückten Lokale-Agenda21-Prozessen ( $\eta=.287 ; \mathrm{p}=.033 ; \mathrm{N}=36)$. Dieser Zusammenhang gilt ebenfalls für den Faktor zivilgesellschaftliches Engagement $(\eta=.113 ; \mathrm{p}=.046 ; \mathrm{N}=36$ ).

Dies deutet darauf hin, dass sich auf Grundlage der analysierten Lokale-Agenda-21-Literatur tatsächlich relevante Faktoren bei der Umsetzung lokaler Nachhaltigkeitsziele ableiten lassen, welche nicht nur tentativ, sondern auch im Rahmen empirischer Überprüfungen eine diskriminative Bedeutung innehaben. Bedeutsame Faktoren wie die finanziellen Ressourcen einer Kommune sowie das zivilgesellschaftliche Engagement der dort lebenden Bürger/innen verdeutlichen, dass

\section{„Durch eine Vernetzung werden erfolgreiche Strategien aus anderen internationalen Lokale-Agenda-21- Kommunen adaptiert."}

es Faktoren gibt, welchen über die Breite an analysierten Studien hinweg eine hohe Relevanz zukommt. Allerdings weisen sie auch auf die hohe Varianz der Lokale-Agenda-21-Prozesse und der vorhandenen Startbedingungen hin, was gleichsam die Vergleichbarkeit der Fälle partiell beschränkt. So geben zwar die identifizierten Faktoren Aufschluss über Optimierungsmöglichkeiten der Lokale-Agenda-21-Prozesse, während gleichsam stets zu berücksichtigen bleibt, dass über potenziell günstige Faktorkombinationen und -wechselwirkungen noch keine Auskunft besteht.

\section{Diskussion}

Die Ergebnisse der durchgeführten Literaturanalyse bestätigen, was Alltagsevidenzen tagtäglich untermauern: Nachhaltigkeit ist ein komplexes Anliegen. Während nachhaltige Entwicklung als Schnittmenge ökologischer, ökonomischer und sozialer Zukunftsfähigkeit bereits als Zieldefinition einen äußerst hohen Interpretations- und Gewichtungsspielraum zulässt, zeigt sich zudem, dass selbst bei bestehender Zielbindung durch konkrete Nachhaltigkeitsprogramme divergierende Ausgangsbedingungen einen hohen Einfluss auf deren tatsächliche Ausgestaltung ausüben.

Dies weist auf eine klassische, im Rahmen der Debatte um die Zuverlässigkeit von Meta- und (systematischen) Literaturanalysen häufig diskutierte Frage hin: Lassen sich die in die Analyse eingegangenen Studien überhaupt vergleichen oder ähnelt ein solcher Versuch nicht einem Vergleich zwischen Birnen und Äpfeln (im Englischen: apples and oranges)? So kritisiert etwa Eysenck (1978, S. 517) am Konzept der Meta-Analyse, dass eine kausale und zielgerichtete Analyse von Studienergebnissen über verschiedene Methoden hinweg als „exercise in mega-silliness" zu bewerten sei. Dagegen wird allerdings schon seit Einführung der meta-analytischen Methodik (durch Glass et al. 1981, Fricke et al. 1985) betont, dass es bei der Integration von Forschungsergebnissen gerade nicht darum geht, identische Untersuchungen zu replizieren, sondern zentrale Hypothesen mit unterschiedlicher Konstruktoperationalisierung zusammenzufassen. Dabei sind Operationalisierungen auf der Seite der abhängigen sowie unabhängigen Variablen zu unterscheiden (Hunter et al. 2014) Bei der abhängigen Variable ist durch die rechtlichen Lokale-Agenda-21-Regelungen die einheitliche Kategorisierung, wenn auch mit inhaltlichen Spezifizierungen, vorgegeben. In Bezug auf die unabhängige Variable 


\section{„Lokale Nachhaltigkeit ist dann erfolgreich, wenn möglichst breite Teile der Bevölkerung sowie der politischen Entscheidungsträger mitwirken."}

ist durch die Unterscheidung von hinderlichen und förderlichen Rahmenbedingungen eine Differenzierung von Oberkategorien vorgenommen, die eine theoretisch wie praktisch sinnvolle Zusammenfassung von Untersuchungen darstellt. Dadurch kann in konstruktiver Zusammenführung der diesbezüglichen Forschungsanstrengungen nachgewiesen werden, dass einige wenige Faktoren wie etwa die finanziellen Ressourcen einer Kommune sowie das zivilgesellschaftliche Engagement der Bürger offensichtlich sowohl über sich inhaltlich und lokal unterscheidende Fälle als auch über methodisch variierende Designs und Auswertungen hinweg als besonders relevante Faktoren zu sichern sind. Die Integration von Untersuchungen mit unterschiedlicher Operationalisierung kann also als ein besonders harter und aussagekräftiger Test auf externe Validität verstanden werden.

Das gilt nicht zuletzt aufgrund der hier vorgelegten Weiterentwicklung des Instruments einer qualitativen Meta-Analyse. Durch den Einsatz der computergestützten (explorativen) Inhaltsanalyse und die große Zahl der einbezogenen Studien wurde ein Gleichgewicht von interpretativem und aggregierendem Vorgehen erreicht. Denn mit diesem Gleichgewicht war eine Frequenzanalyse möglich, die üblicherweise als zentrales Merkmal einer (aggregierenden) quantitativen Inhaltsanalyse gilt (Hunter et al. 2014). Und auf der Grundlage einer solchen Häufigkeitsanalyse sind dann auch (wie demonstriert) Kontingenzanalysen durchführbar, die als Integration des qualitativen und quantitativen Vorgehens angesehen werden können (Ruddat et al. 2010). Das so (fort)entwickelte Konzept einer qualitativen Meta-Analyse empfiehlt sich also gerade auch für systematische Forschungsüberblicke in allen Bereichen der Sozialwissenschaften, in denen es ein Nebeneinander von qualitativen und quantitativen Studien gibt.

In Bezug auf das Ziel der lokalen Nachhaltigkeit können wir deshalb festhalten, dass dieses gesellschaftliche Anliegen hauptsächlich dann erfolgreich ist, wenn möglichst breite Teile der lokalen Bevölkerung sowie der regionalen politischen Entscheidungsträger/innen an dessen Umsetzung mitwirken. Das heißt: Die politischen Akteure sollten erst die förderlichen ökonomischen und politisch-psychologischen Rahmenbedingungen schaffen, damit rechtliche Regelungen in Form der Lokalen Agenda 21 auch wirklich greifen können. Mit dieser Anforderung ergänzen sich prozessbezogene und inhaltliche Ziele der Lokalen Agenda 21 in komplementär-konstruktiver Weise.

\section{Literatur}

Bortz, J./Döring, N. (2007): Forschungsmethoden und Evaluation für Humanund Sozialwissenschaftler: Limitierte Sonderausgabe. Heidelberg, Springer.

Europäische Union (2017): Wie werden die EU-Haushaltsmittel ausgegeben? www.europa.eu/european-union/about-eu/money/expenditure_de

Eysenck, H. J. (1978): An exercise in mega-silliness. In: American Psychologist, 33 (5), 517.

Fricke, R./Treinies, G. (1985): Einführung in die Metaanalyse. Bern, Huber.

Glaser, B./Strauss, A. (1967): Grounded theory: The discovery of grounded theory. In: Sociology. The Journal Of The British Sociological Association, 12, 27-49.

Glass, G. V./McGaw, B./Smith, M. L. (1981): Meta-analysis in social research. Los Angeles: Sage.

Hunter, J. E./Schmidt, F. L. (2014): Methods of meta-analysis, 3. Auflage, Los Angeles: Sage.

Lexikon der Nachhaltigkeit (2015): Lokale Agenda 21. www.nachhaltigkeit. info/artikel/aalborg_chartalokale_agenda_21_651.htm

Moher, D./Liberati, A./Tetzlaff, J./Altman, D. G. (2009): Preferred reporting items for systematic reviews and meta-analyses: the PRISMA statement. In: PLoS Med, 6/7, 264-269.

Nolte, F. (2006): Lokale Agenda 21 zwischen Wunsch und Wirklichkeit: nachhaltige Entwicklung, ihre Aufnahme in Recht und Praxis. Berlin: Duncker \& Humblot.

Teichert, V./Diefenbacher H./Dümig D. (2013): Indikatoren zur Lokalen Agenda 21: ein Modellprojekt in sechzehn Kommunen. Heidelberg: Springer-Verlag.

Ruddat, M ./Schulz, M. (2005): Möglichkeiten und Grenzen einer integrativen Metaanalyse in der Soziologie. In: Historical Social Research 35 (1), 289-322

Rustemeyer, R. (1992): Praktisch-methodische Schritte der Inhaltsanalyse: Eine Einführung am Beispiel der Analyse von Interviewtexten. Münster: Aschendorff.

Steinberg, F./Miranda, L. (2005): Local Agenda 21, capacity building and the cities of Peru. In: Habitat International, 29 (1), 163-182.

Strauss, A. L. (1987): Qualitative analysis for social scientists. Cambridge: Cambridge University Press.

Timulak, L. (2009): Meta-analysis of qualitative studies: A tool for reviewing qualitative research findings in psychotherapy. In: Psychotherapy Research 19 (4/5), 591-600.

Timulak, L./Creaner, M. (2013): Experiences of conducting qualitative metaanalysis. In: Counseling Psychology Review 28 (4), 94-104.

Timulak, L./McElvany, R. (2013): Qualitative meta-analysis of insight events in psychotherapy. In: Counseling Psychology Quarterly 26 (2), 131-150.

Umweltbundesamt (2015): Umweltbewusstsein in Deutschland 2014: Ergebnisse einer repräsentativen Bevölkerungsumfrage. Im Internet: www.umweltbundesamt.de/publikationen/umweltbewusstsein-indeutschland-2014

Walsh, D./Downe, S. (2005): Meta-synthesis method for qualitative research: a literature review. In: J. Adv. Nurs. 50 (2), 204-211.

AUTOR/INNEN + KONTAKT

Norbert Groeben ist Professor für Psychologie an den Universitäten Köln und Heidelberg.

Universität Heidelberg, Psychologisches Institut, Hauptstr. 47, 69117 Heidelberg. Tel.: +49 6221 54-7388, E-Mail: n.groeben@uni-koeln.de

Julia Schnepf ist wissenschaftliche Mitarbeiterin an der Universität Koblenz-Landau.

Universität Koblenz-Landau/Campus Landau, Fortstraße 7, 76829 Landau. E-Mail: schnepf@uni-landau.de

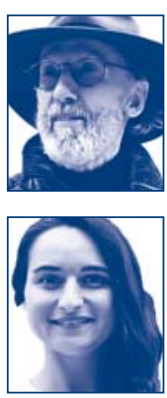

